\title{
Equity in patient experiences of primary care in community health centers using primary care assessment tool: a comparison of rural-to-urban migrants and urban locals in Guangdong, China
}

\author{
Chenwen Zhong ${ }^{1}$, Li Kuang ${ }^{1 *}$, Lina Li ${ }^{1}$, Yuan Liang ${ }^{1}$, Jie Mei ${ }^{1}$ and Li Li ${ }^{2}$
}

\begin{abstract}
Background: The equity of rural-to-urban migrants' health care utilization is already on China's agenda. The Chinese government has been embarking on efforts to improve the financial and geographical accessibility of health care for migrants by strengthening primary care services and providing universal coverage. Patient experiences are equally vital to migrants' health care utilization. To our knowledge, no studies have focused on equity in the patient experiences between migrants and locals. Based on a patient survey from Guangdong, China, which has a large number of rural-to-urban migrants, our study assessed the equity in the primary care patient experiences between rural-to-urban migrants and urban locals in the same health insurance context, since different forms of insurance can affect the patient experiences of primary care.

Methods: We stratified our samples by different insurance types into three layers. We assessed primary care patient experiences using a validated Chinese version of the Primary Care Assessment Tool (PCAT), including eight primary care attributes. A 'PCAT total score' was calculated. Data were collected through face-to-face and one-on-one surveys in 2014. Propensity score matching (PSM) was used for each layer to generate comparable samples between rural-to-urban migrants and urban locals. Based on the matched dataset, a t-test was employed to compare the primary care patient experiences of the two groups.

Results: Using PSM, 220 patients in the rural-to-urban migrants group were matched to 220 patients in the urban locals group. After the matching, the observed confounding variables were balanced, and the PCAT scores were almost equal between the two groups. The only slight differences existed in the Urban Employee Basic Medical Insurance layer and in the without basic medical insurance coverage layer.

Conclusions: Equity in the primary care patient experiences between rural-to-urban migrants and urban locals seems to have been achieved to some extent. However, there is room for improvement in the equity of coordination of care and comprehensiveness. Policy makers should consider strengthening these two dimensions by integrating the health care system. More attention should be focused on helping migrants break down language and cultural barriers and improving the patient-physician communication process.
\end{abstract}

Keywords: Equity, Patient experiences, Primary care, Rural-to-urban migrants, China

\footnotetext{
* Correspondence: kuangli@mail.sysu.edu.cn

'Department of Health Administration, School of Public Health, Sun Yat-sen

University, Guangzhou 510080, China

Full list of author information is available at the end of the article
}

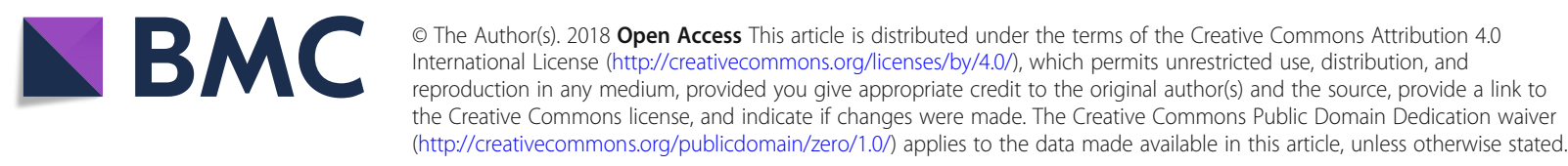




\section{Background}

Health equity refers to an absence of disparities in health care or its socio-determinants between groups within different socioeconomic classes or resulting from social, political, economic or other factors that might have an effect on health care status and equity [1-3]. An important social determinant of health care in China is the long existing household registration ('hukou') system, which was implemented in 1950s and categorizes people into urban and rural [4] groups; this is a very strong determinant of the rights and privileges affecting socioeconomic wellbeing [5]. When rural people migrate to search for jobs in urban areas, it is never easy for them to convert their household registration to an official urban residency [6]. Since China's public policies have long been introduced based on this 'hukou' system, rural-to-urban migrants have always been identified as being vulnerable due to their poor living conditions [7], lack of social support [8], lack of health risk awareness [9], high medical costs [10, 11] and limited access to health care [12], jobs and insurances [13, 14]. In 2015, the number of migrants in China had reached approximately 247 million, which accounts for $18 \%$ of the total Chinese population [15]. Guangdong Province in southeastern China accounts for a large amount of migration, especially in the several cities in the Pearl River Delta region [16]. The increasing number of rural-to-urban migrants and their perception of the fairness of the health care system has caused a great deal of concern for most governments and citizens.

To decrease the disparities between rural-to-urban migrants and urban locals, China has been embarking on health care reform to provide equal access and affordable healthcare for all by 2020 [17]. One of the important measures is the strengthening of primary care, starting with increasing the number of and funding for community health centers (CHCs) in urban areas to make health care services more accessible and less expensive [18]. Being the most financially and geographically accessible approach to health care, primary care provides access to rural-to-urban migrants to obtain health care and reduces the socioeconomic and geographic disparities among different groups $[19,20]$. Another goal of China's current healthcare reform is to establish universal insurance coverage for the whole population. Since the New Rural Cooperative Medical System has been merged into the Urban Resident Basic Medical Insurance in most developed areas, including in Guangdong Province since 2014 [21], the current health care system includes Urban Resident Basic Medical Insurance (URBMI) and Urban Employee Basic Medical Insurance (UEBMI). Financed by employers and employees, migrant workers employed in urban formal sectors, including state-owned, collective, private enterprises and NGOs, are eligible for UEBMI. In some cities, UEBMI also covers part-time workers. The funds are managed to cover outpatient and inpatient services [22]. Migrants who work in urban informal sectors or who are unemployed or self-employed can enroll in URBMI on an individual basis, which is financed mainly by individuals, with few government subsidies. URBMI covers hospital care and catastrophic illness [23]. Evidence has shown that medical health insurance provides positive financial protection for migrants regardless of the type of scheme [22, 24].

All of these efforts can improve patients' utilization of the health care system by improving geographical and financial access. Furthermore, the patient experience is another important determinant of health care utilization; it may receive comparatively less attention in the literature but is of central importance to patients' future health care-seeking behavior. According to The Beryl Institute, patient experience is defined as the sum of all interactions shaped by an organization's culture that influence patient perceptions across the continuum of care [25]. Evidence has shown that how patients perceive the process of consultation may influence their future decisions about seeking care [26]. Saether and colleagues [27] investigated migrants' access to antiretroviral therapy in Thailand and found that participants experienced discrimination such as rude doctors and challenges accessing health care a second time. Another study in Poland found that when migrants felt a general sense of insecurity about the host area's health care system, either due to misunderstandings or feeling disregarded by the doctors for being migrants, they might change their health care-seeking strategy, stop visiting doctors in the host area and try to consult doctors in their familiar homeland [28]. In other words, even though equity in financial and geographical accessibility has been achieved, equity in the patient experience could be a significant factor influencing patients' health care utilization.

We are aware that patient experiences in primary care can be contextualized to measure primary care's five core dimensions (first-contact utilization, first-contact accessibility, continuity, coordination, and comprehensiveness) and three derivative dimensions (family centeredness, community orientation, and cultural competence). This approach has been widely used in recent years, because it directly describes the primary care process [29-32]. Firstcontact utilization measures the extent to which primary care acts as an entry point for other levels of care, whereas first-contact accessibility measures the extent to which patients are able to access primary care for each new problem. Continuity of care refers to the longitudinal use of a usual source of care over time. Coordination of care is the linking of health care visits and services between different levels of care so that patients can receive appropriate care and meet health needs. Comprehensiveness of care refers 
to the availability of a wide range of services in primary care and their appropriate provision. Family centeredness reflects the participation of family in the assessment and treatment of a patient. Community orientation measures providers' knowledge of community health care needs. Cultural competence measures whether the patient recommends the primary care provider to others [33, 34]. In China, rural-to-urban migrants experience less satisfaction in terms of the types of drugs available, attitudes of health workers, and waiting times than local residents in community health centers [35]. Their experiences of primary care did not show higher scores in any of the domains mentioned above in CHCs than in tertiary hospitals [36].

Studies have focused on equity in primary care experiences between groups with different levels of social disadvantage, such as health status [37], household income [38], age [39], race and ethnicity [40], and disability [41]. People with high self-ratings of health scored higher in the family/community orientation dimension and total primary care scores [37]. The higher household income group was more likely to experience better primary care, such as comprehensiveness [38]. Older individuals reported significantly better experiences across many dimensions of primary care, such as first-contact utilization and continuity [39]. However, no studies have focused on equity in patient experiences of primary care between rural-to-urban migrants and urban locals. Using a patient perception primary care assessment survey [the Primary Care Assessment Tool (PCAT)] administered in 2014, we aimed to determine whether the patient experience of primary care is equal between rural-to-urban migrants and urban locals.

Since medical health insurance can influence patients' health seeking behavior and affect patients' health care utilization $[42,43]$, we first stratified our data into three layers based on the different medical health insurance conditions, including UEBMI, URBMI and individuals without basic medical insurance coverage (WBMI). According to our previous studies, patients' socioeconomic characteristics have a significant impact on their primary care experiences [44], which may limit the extent to which household registration results in equal access to health care. To adjust for confounding variables and improve causal inferences, we conducted propensity score matching (PSM) within each insurance layer to generate comparable samples between the rural-to-urban migrants and urban locals. PSM has been widely used in primary care observational studies [45-47]. After matching, we compared the patient experiences of the rural-to-urban migrants to the urban locals within each layer. It is also hoped that policy implications regarding the management of issues related to ruralto-urban migrants can be drawn from the analysis.

\section{Methods \\ Study design}

This study was a cross-sectional survey conducted in Guangdong Province, southern China, due to its relatively large and diverse population. A stratified, three-stage sampling approach was used to determine the study sample. In the first stage, we selected three cities (Guangzhou, Dongguan, and Shenzhen), which were the most popular metropolitan areas and attracted millions of rural laborers to work as nonpermanent migrants in the Pearl River Delta region in southern China [48]. In the second stage, eight $\mathrm{CHCs}$ were selected typically on the basis of their representativeness in terms of the socio-demographic, environmental, and health coverage factors in the area. In the third stage, patients in each $\mathrm{CHC}$ were selected by convenience sampling to participate in our survey. $\mathrm{Pa}-$ tients who were 18 years or older, could speak Mandarin or Cantonese, and visited the same general practitioners at least three times were selected, since they were considered to have a better understanding of primary care services. Those who were visiting general practitioners were excluded. Based on the standard sample size formula for a cross-sectional study, a target sample size of 680 was set for each group given a type I error of 0.05 , type II error of 0.1 , and refusal rate of $10 \%$ [49].

\section{Instrument}

We used a validated Chinese version of the PCAT to evaluate patient primary care experiences and service delivery [49-51]. Following the original edition of the PCAT [33], an assessment of each subdomain was obtained from the survey subjects' responses to each question using a 4-point Likert-type scale of 1 (definitely not), 2 (probably not), 3 (probably), and 4 (definitely). Additional response options included 'not sure' or 'don't know', which were coded as the middle score (2.5). A 'PCAT total score' was created by summing the scores of the eight essential dimensions to summarize the overall primary care experience. Our questionnaire consisted of 42 items. A total of 23 items were used to assess the five core dimensions and three derivative dimensions of primary care. Two items were used to identify whether the patients has usual sources of care, two items measured the frequency of visits to primary care practitioners, one item assessed the patients' global satisfaction with their current health care provider, and the remaining items were mainly used to reflect the patients' socioeconomic characteristics, including gender, age, occupation, education, income, marital status, health status, chronic disease status, and social medical insurance status.

\section{Data collection}

Data collection began in June and continued to August 2014. Postgraduate students from Sun Yat-Sen University were hired as interviewers and had at least 6 months' prior 
experience working as interviewers. All interviewers were trained by the principal investigator (LK), who was involved in the pilot study, to assist patients to complete the questionnaires, and the interviewers had daily supervision from senior researchers (YL and JM). Individual face-toface interviews were conducted in the waiting area. The selected patients were asked for permission to participate in our study with a full explanation of the purpose and were told that the survey would not influence their GP visits. After the interview, a small gift was given to patients who took part in the survey to show our gratitude for completing the questionnaire.

\section{Data analysis}

The final sample recruited consisted of 1461 patients. First, we stratified our data into three layers based on the different medical health insurance conditions, including UEBMI (locals: 506, migrants: 121), URBMI (locals: 190, migrants: 334), and WBMI (locals: 19, migrants: 291). Then, we employed PSM within each insurance layer through a nearest neighbor matching algorithm with a match tolerance of 0.1. Finally, we obtained 220 pairs of samples after matching (120 pairs for UEBMI, 82 pairs for URBMI, and 18 pairs for WBMI).

Continuous variables were reported as the mean \pm standard deviation, and categorical variables were reported as frequency (\%). A chi-square test was used to study any differences between the two groups of categorical data. Based on the matched dataset, the PCAT scores were compared with respect to household registration within each layer using a t-test. The level of significance was $p<0.05$. All statistical analysis was performed using the statistical package IBM SPSS23.

\section{Results}

\section{Descriptive statistics}

A total of 1461 participants were eligible for inclusion in our study. A total of 220 patients in the rural-to-urban migrant group were matched with 220 patients in the urban local group after propensity score matching. Table 1 shows the characteristics of the entire study sample for urban locals and rural-to-urban migrants before and after the PSM. Before the PSM, 51\% of the respondents were rural-to-urban migrants. In both groups, most respondents were female, retired and unemployed. Though the majority of respondents in both groups were 31 to 60 years old, the percentage of rural-to-urban migrants aged $>60$ was only $5.0 \%$ while for urban locals it was $43.3 \%$. A majority of the respondents stated that their health status was fair or poor $(71.8 \%$ of the ruralto-urban migrants and $82 \%$ of the urban locals). While $60.6 \%$ of the urban locals had at least one chronic disease, $74 \%$ of the rural-to-urban migrants were without chronic diseases. Most respondents in both groups had not contracted with a primary care physician (PCP). Of the rural-to-urban migrant respondents, 39\% were without medical insurance. Compared to the urban locals (only $2.6 \%$ of whom were without medical insurance), this proportion is very high.

There were statistically significant differences in age, employment status, education, marital status, health status, chronic disease condition, number of $\mathrm{CHC}$ visits in the last year, whether they had contracted with a PCP or not and social medical insurance between the two groups before the PSM $(p<0.05$, Table 1$)$. We found that after matching all the confounding variables observed, the patient characteristics in each layer were balanced $(p>0.05)$. An additional file shows this in greater detail [see Additional file 1].

\section{Propensity score matching (PSM) results}

The propensity score was constructed using common logistic regression modeling in which potential confounding variables were considered independent variables, including gender, age, employment status, household income, education, marital status, health status, chronic disease status of respondents, the number of community health center visits in the last year and whether respondents had contracted with a PCP or not. The group assignment (rural-to-urban migrants vs. urban locals) was included as the dependent variable. Figure 1 shows the distribution of propensity scores between the two groups before and after matching within the three layers. We can easily determine that after the PSM, the distribution of biases and confounding variables between the two groups was almost balanced.

\section{Primary care dimension scores}

Table 2 presents the primary care assessment scores between migrants and urban locals in each insurance layer respectively, before and after the PSM. The total scores of rural-to-urban migrants in different insurance statuses ranged from 13.63 to 13.68 , while the scores of urban locals ranged from 13.47 to 13.49 before the PSM. After the matching, while other scores may have had small fluctuations, only the score of migrant patients who had no health insurance coverage changed significantly from 13.64 to 12.63 .

After the PSM, the data from the two groups were balanced by socioeconomic characteristics and health service utilization patterns. In the UEBMI layer, rural-to-urban migrants reported slightly higher scores for the attributes of first-contact accessibility, coordination, family centeredness, community orientation, cultural competence and total score. A significant difference was only reported for coordination of care $(p=0.002)$. In the WBMI layer, urban locals reported a higher score for the attribute of comprehensiveness $(p=0.047)$. In the URBMI layer, there turned 
Table 1 Comparability of socioeconomic characteristics and health care utilization patterns by group before and after PSM

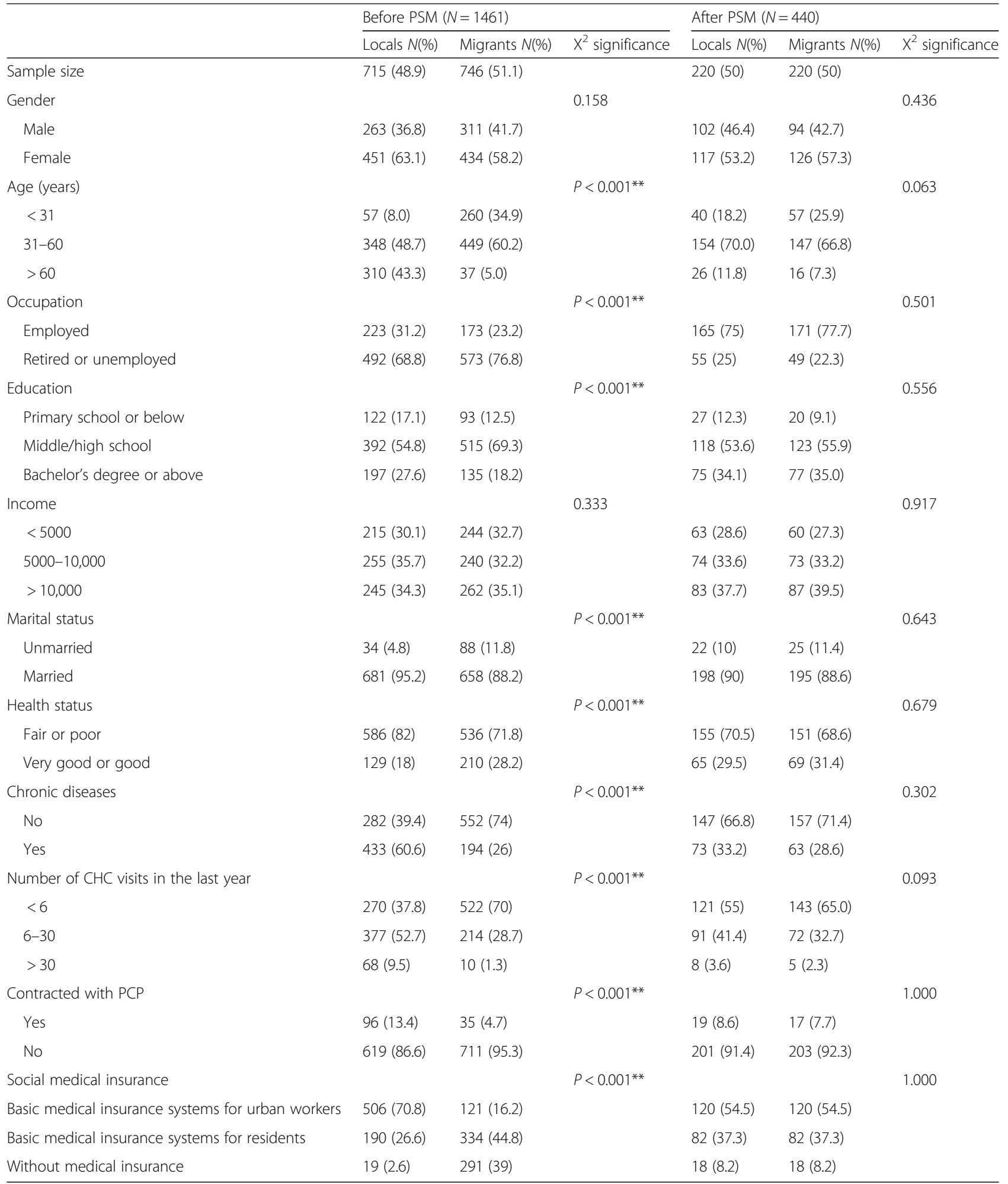

Note:1. $N$ number of patients; ${ }^{*} p<0.05 ;{ }^{* *} p<0.01 ; P S M$ propensity score matching, $P C P$ primary care physician

2. Differences were explored using the chi-square test between urban locals (with 'hukou') and rural-to-urban migrants (without 'hukou') who settled permanently or temporarily somewhere other than the original household registration location before and after propensity score matching 

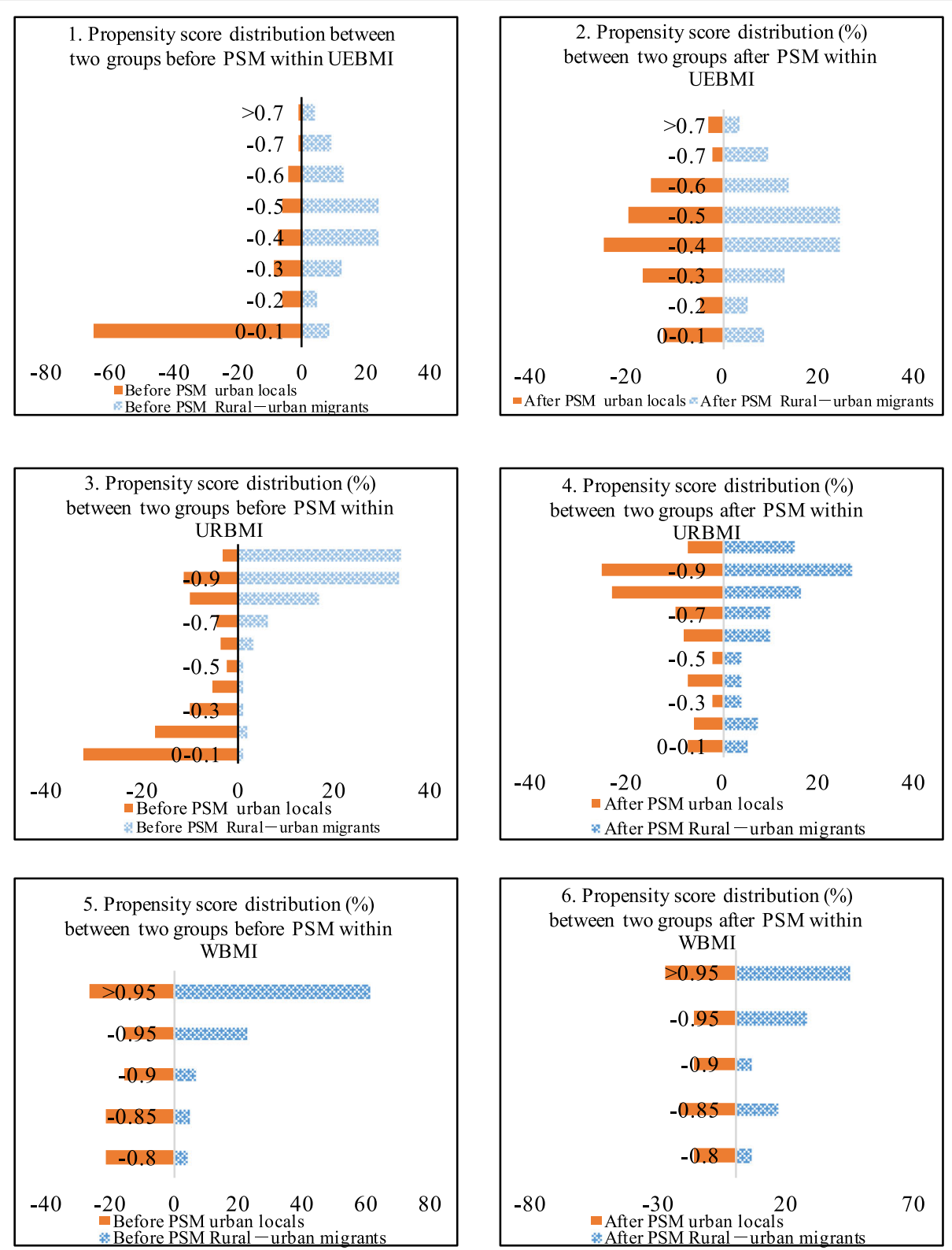

Fig. 1 Distributions of the propensity scores between two groups before and after matching within each layer

out to be no difference between rural-to-urban migrants and urban locals (Table 2, Figs. 2, 3 and 4).

\section{Discussion \\ Main finding}

In this study, we compared rural-to-urban migrants' primary care experience to that of urban locals' in the same insurance context after PSM using a validated Chinese edition of the PCAT. The findings demonstrated that patient primary care experiences between rural-to-urban migrants and urban locals were almost equal within the same health insurance layer after using PSM. However, there was still a small difference in the perception of the coordination of care within the UEBMI layer and the perception of comprehensiveness within the WBMI layer between the two groups.

In our study, the PCAT scores for the eight attributes and the total scores were almost equal for both groups (rural-to-urban migrants and urban locals). This implies that regardless of the household registration with which patients are associated, their experiences of primary care are the same. Li [35] conducted a survey to compare patients' perceived quality of care among migrants and local patients using patient satisfaction as an indicator and reached 


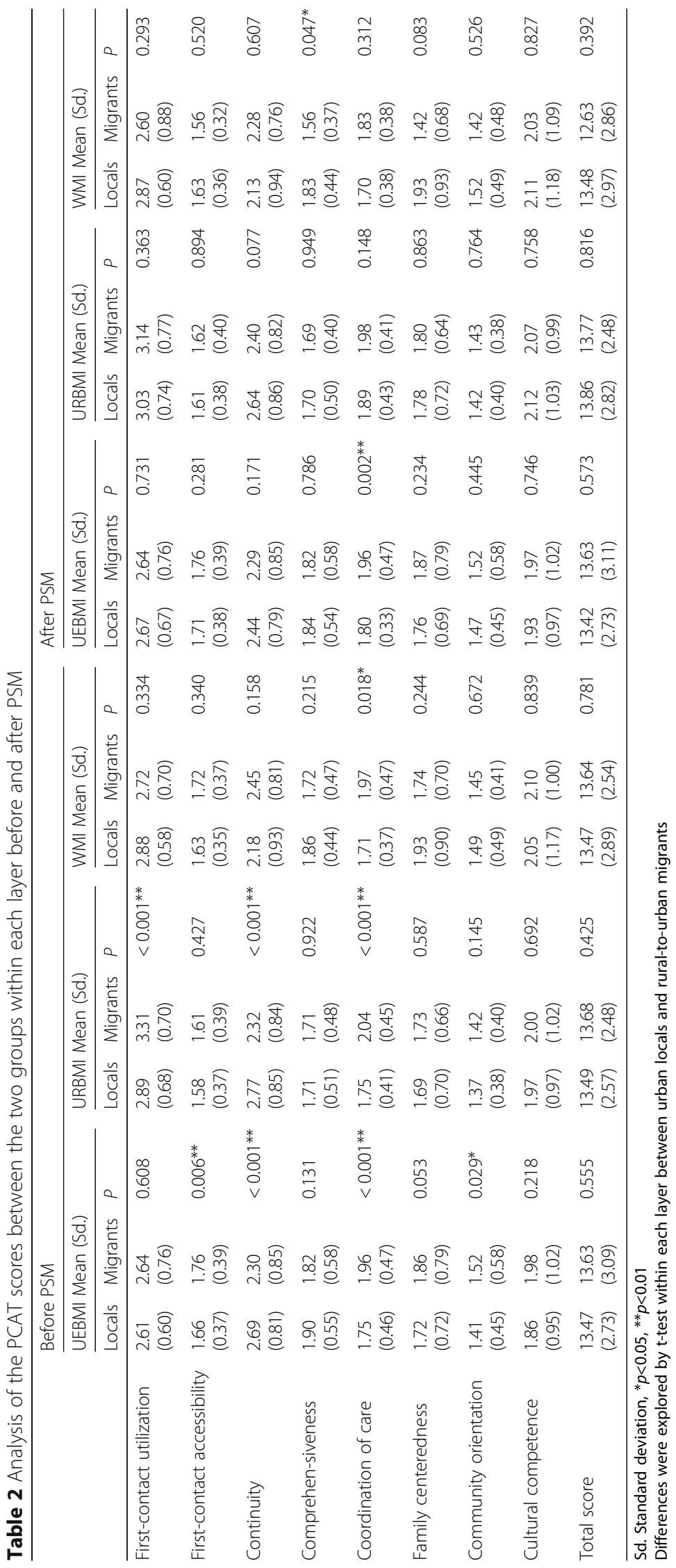




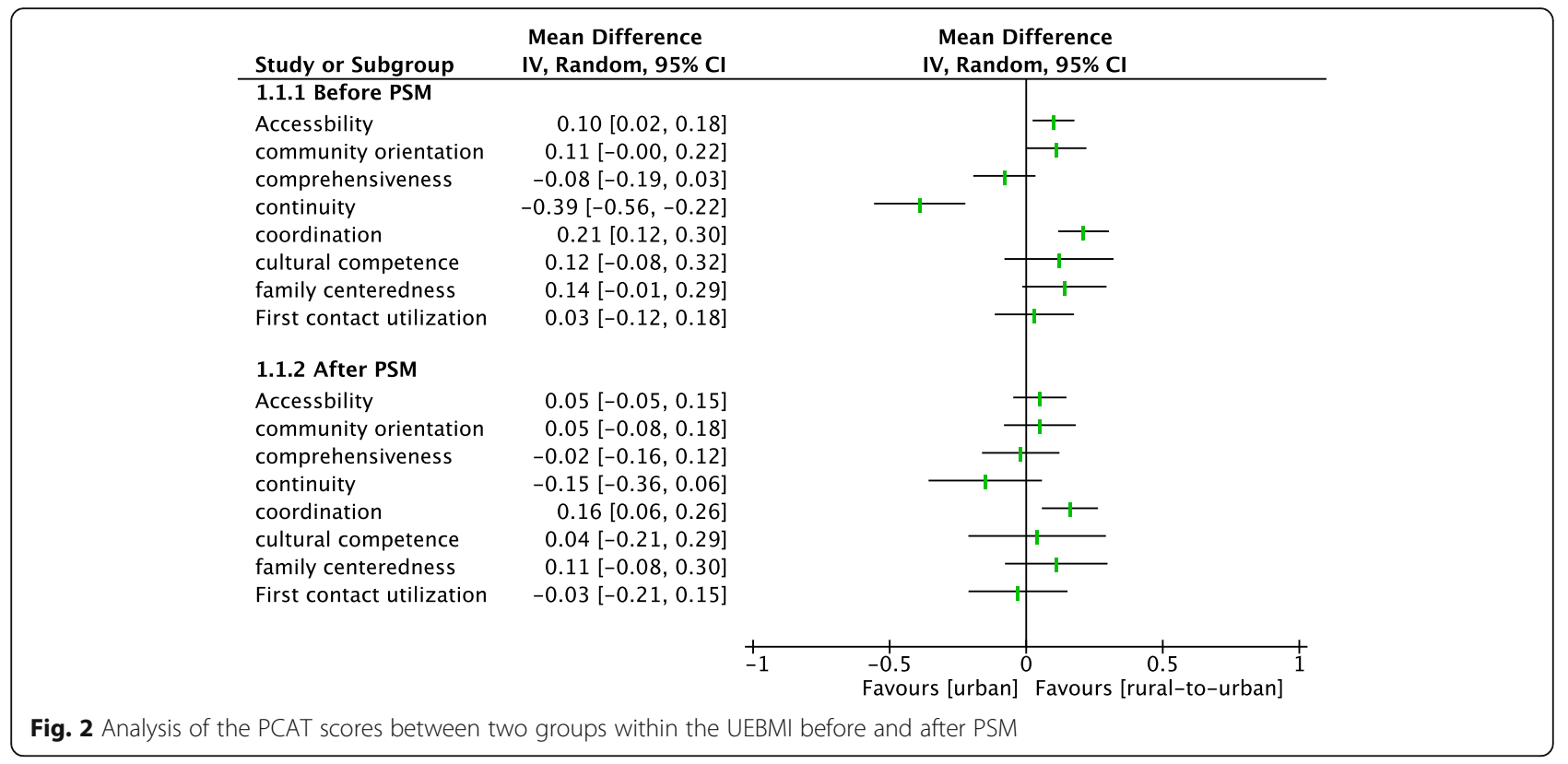

a similar conclusion, with no difference in the overall satisfaction between the local residents and migrants. Over the past decades, health care reform has ensured affordable access to health care services with the achievement of nearly universal health insurance coverage and rapidly developing primary care. The results from our study demonstrate that China's health reform may have to some extent achieved the goal of equity in patients' experiences of health care between migrants and locals.

Nevertheless, there were still small differences in the UEBMI layer and the WBMI layer between the two groups. Since we have employed PSM to improve the comparability of the two groups, theoretically, these differences were not caused by socioeconomic status but by household registration.

One surprising finding was that the vulnerable ruralto-urban migrants $(1.96 \pm 0.47)$ attributed a substantially higher score than the urban locals $(1.80 \pm 0.33)$ to the coordination of care within the UEBMI layer. When we then compared the items under coordination of care, we found that among the patients with UEBMI coverage, the item "whether primary physicians would recommend specialists for you when you need a referral" in the

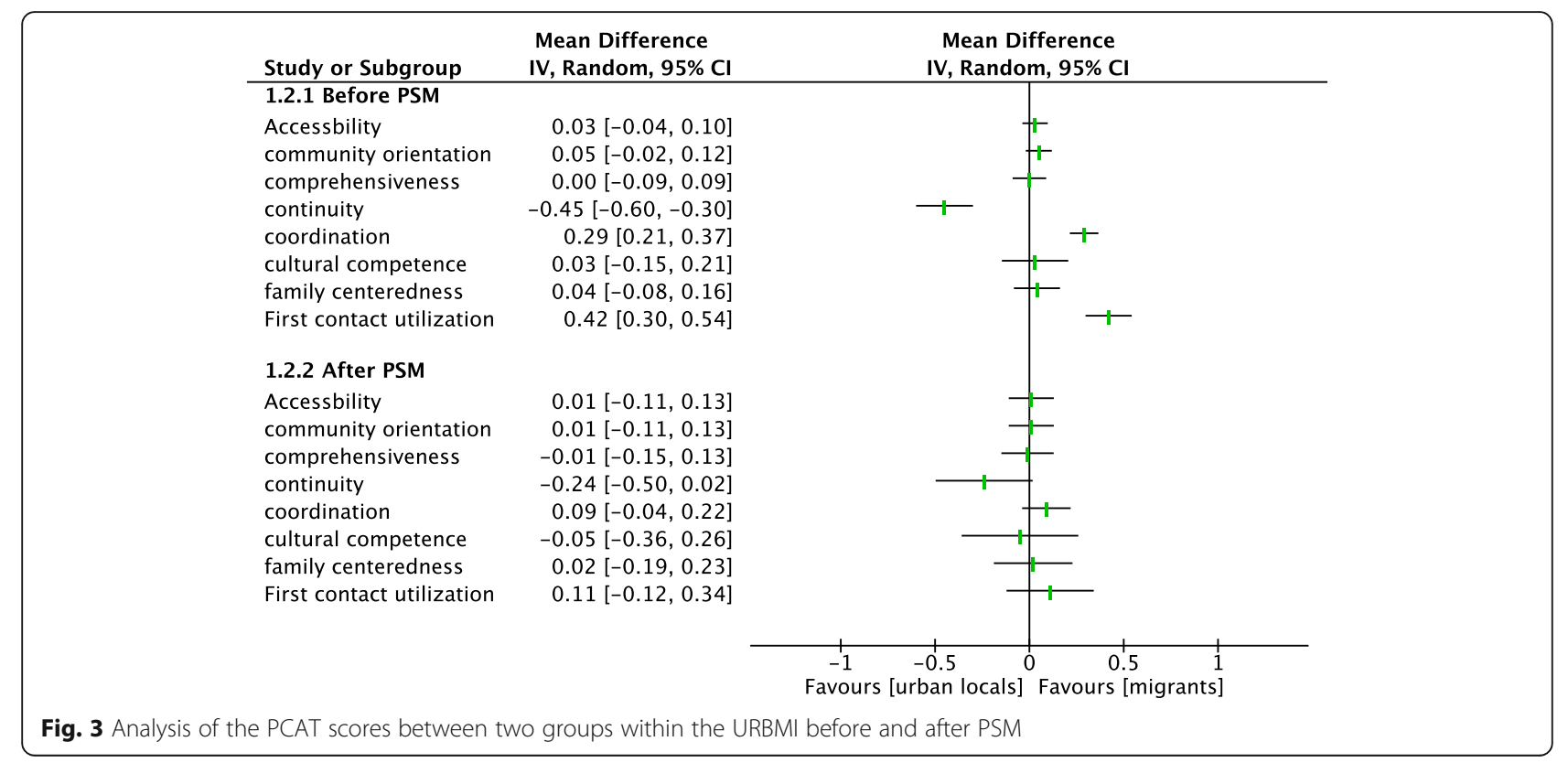




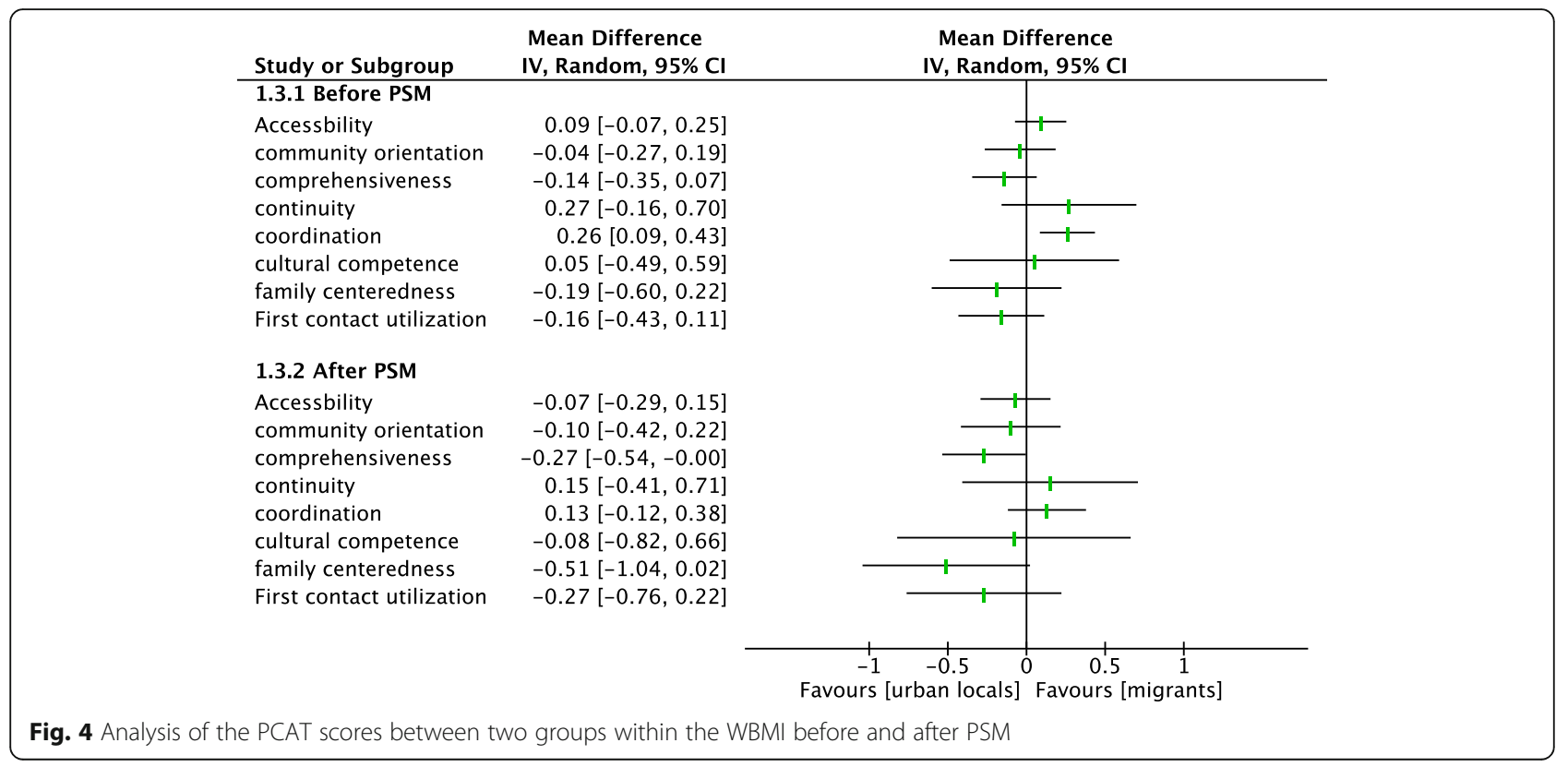

coordination domain scored significantly higher than for urban locals $(1.74 \pm 0.98$ for rural-to-urban migrants, 1.29 \pm 0.60 for urban locals, $P=0.003$ ). In moving from the rural to the urban context, migrants' social support network was fragmented. Research has shown that migrants consider PCPs to be more familiar with their health condition [36]. When they must seek health care from specialists, they might be prone to go to primary health centers for help. Yang et al. [17] revealed that patients in Chinese urban areas did not generally trust community health service centers. This belief may result in urban patients choosing to see specialists in hospitals when needed, which weakens the use of appropriate referrals by PCPs.

In our study, of the 746 migrants surveyed, 291 had no health insurance. In the local group, only 19 (of 715) patients were not covered by health insurance. These data mainly reflected the fact that migrants were still largely excluded from medical benefits in urban cities. For these patients' WBMI coverage, urban locals gave higher scores than rural-tourban migrants to the attribute of comprehensiveness. The comprehensiveness of primary care measures the ability of physicians to provide a wide range of care in response to patients' health needs [19]. While providing comprehensiveness, the health care provider's accumulated knowledge of the patient could be directly affected by patient-physician communication [52]. Compared to urban locals, some of the migrants might have trouble communicating to physicians due to language barriers and a lack of family support. Cleland found that physicians might need to make a greater effort to understand migrant patients because of language and cultural barriers [53]. Therefore, for urban locals, language familiarity and a better understanding of the health care system may be important facilitators of their ability to communicate more easily with health service providers and receive more comprehensive care.

\section{Limitations}

There are several limitations to this study. First, our findings might overestimate the real situation of health equity between rural-to-urban migrants and urban locals since our survey was conducted among patients who have consulted at $\mathrm{CHCs}$ at least once in a year. Patients who felt discriminated against or who once had an unsatisfactory experience in the $\mathrm{CHCs}$ would have been excluded in our study. Second, we did not investigate patient primary care experience in health care settings other than the $\mathrm{CHCs}$, though the $\mathrm{CHCs}$ are becoming the major primary care providers in China. Participants could also experience primary care in tertiary hospitals, and so any implications of the findings should be tempered with a word of caution. Third, the sample of local patients without medical health insurance coverage included 19 respondents, which was a relatively small sample. After the PSM, there were only 18 pairs of samples in the WBMI layer, which lacked enough power to detect intervention effects. Despite all of these limitations, to our knowledge, our study is the first in China to address the equity of patient primary care experiences between rural-to-urban migrants and urban locals covered by the same insurance, and it provides significant evidence for health care policy in China. 


\section{Conclusions}

Our study demonstrates that rural-to-urban migrants receiving primary care from $\mathrm{CHCs}$ reported equal primary care experiences when compared to urban locals in the same medical health insurance context. The hope of health care equity between rural-tourban migrants and urban locals seems to have been achieved in the $\mathrm{CHCs}$ to some extent. However, there is room for improvement in the equity of coordination of care and comprehensiveness. Policy makers should consider strengthening coordination of care and comprehensiveness in primary care by integrating health care systems. More attention should be focused on helping migrants break down language and cultural barriers and improving the patient-physician communication process.

\section{Additional file}

Additional file 1 Comparability of socioeconomic characteristics and health care utilization patterns by group based on different health insurance schemes before and after PSM. (DOCX $30 \mathrm{~kb}$ )

\section{Abbreviations}

CHCs: community health centers; PCAT: Primary Care Assessment Tool; PSM: propensity score matching; UEBMI: Urban Employee Basic Medical Insurance; URBMI: Urban Resident Basic Medical Insurance; WBMI: without basic medical insurance coverage

\section{Acknowledgements}

The authors thank all of the health care staff from the community health centers in Guangzhou, Dongguan, and Shenzhen in Guangdong Province for their enormous support on-site during the data collection.

\section{Funding}

This study was supported by the National Natural Science Foundation of China (Grant No: 71673311) and the Health and Family Planning Commission of Guangdong Province.

\section{Availability of data and materials}

Please contact the corresponding author for data requests.

\section{Authors' contributions}

LK and CWZ conceived of the study. LK provided overall guidance including determining the study design and instrument development. $\mathrm{YL}$ and $\mathrm{JM}$ collected the data. CWZ, LNL, YL, and JM were involved in data cleaning. CWZ, LK and LNL conducted the data analysis. CZW, LK, $\mathrm{LNL}$ and $\mathrm{LL}$ contributed to the literature search and the interpretation of the data. CWZ wrote the first draft. LK and LL contributed feedback on the study results and approved the final version of the manuscript submitted for publication.

\section{Ethics approval and consent to participate}

Informed oral consent was obtained from all participants. Ethical approval for the study was obtained from the Institutional Review Board of the School of Public Health, Sun Yat-Sen University, P.R. China. The protocol for this study conforms to the guidelines of the 1975 Declaration of Helsinki.

\section{Competing interests}

The authors declare no conflicts of interest.

\section{Publisher's Note}

Springer Nature remains neutral with regard to jurisdictional claims in published maps and institutional affiliations.

\section{Author details}

${ }^{1}$ Department of Health Administration, School of Public Health, Sun Yat-sen University, Guangzhou 510080, China. ${ }^{2}$ Department of Family Medicine and Community Health, Case Western Reserve University, Cleveland, $\mathrm{OH} 44106$, USA.

Received: 21 December 2017 Accepted: 10 April 2018 Published online: 27 April 2018

\section{Reference}

1. Whitehead M. The concepts and principles of equity and health. Int J Health Serv. 1992:22:429-45.

2. Braveman P, Gruskin S. Defining equity in health [J]. J Epidemiol Community Health. 2003;57(4):254.

3. Marmot M, Friel S, Bell $R$, et al. Closing the gap in a generation: health equity through action on the social determinants of health [J]. Lancet. 2009;372(9650):1661-9.

4. Yang DT, Zhou H. Rural-urban disparity and sectoral labor allocation in China. J Dev Stud. 1999;35(3):105-33.

5. Sun M. The Potential Causal Effect of Hukou on Health among Rural-toUrban Migrants in China. 2015. [Thesis].

6. Yang DT, Zhou H. Rural-urban disparity and sectoral labour allocation in China [J]. Working Papers. 1999;35(3):105-33.

7. Lu L, Zou G, Zeng Z, et al. Health-related quality of life and its correlates among Chinese migrants in small- and medium-sized Enterprises in two Cities of Guangdong [J]. PLoS One. 2014;9(1):e83315.

8. Li Y. Understanding health constraints among rural-to-urban migrants in China [J]. Qual Health Res. 2013;23(11):1459.

9. Shi $Y$, Ji $Y$, Sun J, et al. Lack of health risk awareness in low-income Chinese youth migrants: assessment and associated factors [J]. Environ Health Prev Med. 2012;17(5):385-93.

10. Inoue M, Kachi Y. Should co-payments for financially deprived patients be lowered? Primary care physicians' perspectives using a mixedmethods approach in a survey study in Tokyo [J]. Int J Equity Health. 2017;16(1):38.

11. Hu X, Cook S, Salazar MA. Internal migration and health in China. Lancet. 2008:372(9651):1717-9.

12. Hong Y, Li X, Stanton B, et al. Too costly to be ill: healthcare access and health-seeking Behaviours among rural-to-urban migrants in China [J]. World Health Popul. 2006:8(2):22.

13. Peng $Y$, Chang $W$, Zhou $H$, et al. Factors associated with health-seeking behavior among migrant workers in Beijing, China [J]. BMC Health Serv Res. 2010;10(1):1-10.

14. Zhang A, Treiman DJ. Social origins, hukou conversion, and the wellbeing of urban residents in contemporary China. Soc Sci Res. 2013; 42:71-89.

15. Department of Migrant Population, Nation Health and Family Planning Commission, China. 2016 Report on China's migrant population development. Beijing, China: China Population Press. p. 2016.

16. Chen $X Z$. Studies on the urban living willingness of the floating population in Guangdong province [D]. Jilin Province: JiLin Province: JiLin University, 2017 (in Chinese)

17. Yang Y, Yang D. Community health service centers in China, not always trusted by the populations they serve? [J]. China Econ Rev. 2009;20(4): $620-4$.

18. Wang $\mathrm{Y}$, Wilkinson $\mathrm{M}, \mathrm{Ng}$ E, Cheng KK. Primary care reform in China. Br J Gen Pract. 2012:62:546-7.

19. Starfield B. Primary care: an increasingly important contributor to effectiveness, equity, and efficiency of health services. SESPAS report 2012 [J]. Gac Sanit. 2012:26(1):20-6

20. Ferrer RL, Hambidge SJ, Maly RC. The essential role of generalists in health care systems. [J]. Annals of Internal Medicine. 2005;142(8):691.

21. State Council of the People's Republic of China. Opinions of the State Council on the establishment of a unified basic pension insurance system for urban and rural residents. 2014

22. Chen W, Zhang $\mathrm{Q}$, Renzaho AMN, et al. Social health insurance coverage and financial protection among rural-to-urban internal migrants in China: evidence 
from a nationally representative cross-sectional study []]. Bmj Global Health. 2017;2(4):e000477.

23. Qin X, Pan J, Liu GG. Does participating in health insurance benefit the migrant workers in China? An empirical investigation [J]. China Econ Rev. 2014;30:263-78.

24. Cheng SH, Chiang TL. The effect of universal health insurance on health care utilization in Taiwan. Results from a natural experiment [J]. Jama. 1997; 278(2):89.

25. Niederhauser V, Wolf J, Marshburn D, et al. Defining Patient Experience[J]. Patient Experience Journal. 2014;1:7-19.

26. Murray J, Corney R. Not a medical problem? An intensive study of the attitudes and illness behaviour of low attenders with psychosocial difficulties [J]. Soc. Psychiatry Psychiatr. Epidemiol. 1990;25(3):159.

27. Saether S, Chawphrae UM, Keizer C, et al. Migrants' access to antiretroviral therapy in Thailand [J]. Tropical Med Int Health. 2010;12(8):999-1008.

28. Anna CE, Mette S. "Where to find those doctors?" A qualitative study on barriers and facilitators in access to and utilization of health care services by Polish migrants in Norway [J]. Bmc Health Services Research. 2016;16(1):460.

29. Wang $H H$, Wong $S Y$, Wong MC, et al. Attributes of primary care in community health centres in China and implications for equitable care: a cross-sectional measurement of patients' experiences []]. Qjm Monthly J. Assoc. Physicians. 2015; 108(7):549.

30. Mccollum $R$, Chen $L$, Tang $C X$, et al. Experiences with primary healthcare in Fuzhou, urban China, in the context of health sector reform: a mixed methods study []]. International Journal of Health Planning \& Management. 2014:29(2):e107-26.

31. Lee JH, Choi YJ, Sung NJ, et al. Development of the Korean primary care assessment tool measuring user experience: tests of data quality and measurement performance [J]. Int J Qual Health Care. 2009;21(2):103-11.

32. Wong SY, Kung K, Griffiths SM, et al. Comparison of primary care experiences among adults in general outpatient clinics and private general practice clinics in Hong Kong [J]. BMC Public Health. 2010;10:397.

33. Shi L, Starfield B, Xu J, et al. Validating the adult primary care assessment tool [j]. J Fam Pract. 2001;50(2):161.

34. John Hopkins Bloomberg School of Public Health. 2010. Primary care assessment tools. [Online] https://www.jhsph.edu/research/centers-andinstitutes/johns-hopkins-primary-care-policy-center/pca_tools.html.

35. Li H, Chung RY, Wei X, et al. Comparison of perceived quality amongst migrant and local patients using primary health care delivered by community health centres in Shenzhen, China [J]. BMC Fam Pract. 2014;15(1):76.

36. Zeng J, Shi L, Zou X, et al. Rural-to-urban Migrants' experiences with primary care under different types of medical institutions in Guangzhou, China [J]. PLoS One. 2015;10(10):e0140922.

37. Sung NJ, Markuns JF, Park KH, et al. Higher quality primary care is associated with good, self-rated health status [J]. Fam Pract. 2013;30(5):568-75.

38. Owolabi O, Zhang Z, Wei X, et al. Patients' socioeconomic status and their evaluations of primary care in Hong Kong [J]. BMC Health Serv Res. 2013; 13(1):1-11.

39. Dahrouge $S$, Hogg W, Tuna $M$, et al. Age equity in different models of primary care practice in Ontario [J]. Can. Fam. Physician. 2011;57(11):1300-9.

40. Stevens GD, Shi L. Racial and ethnic disparities in the quality of primary care for children. [J]. Journal of Family Practice. 2002;51 (6):573.

41. Almeida MHMD, Pacheco S, Krebs S, et al. Primary health care assessment by users with and without disabilities [J]. Codas. 2017;29(5):e20160225.

42. Inoue M, Kachi Y. Should co-payments for financially deprived patients be lowered? Primary care physicians' perspectives using a mixed-methods approach in a survey study in Tokyo. []]. International Journal for Equity in Health. 2017;16(1):38

43. Liu X, Wong H, Liu K. Outcome-based health equity across different social health insurance schemes for the elderly in China [J]. BMC Health Serv Res. 2015;16(1):1-12

44. Kuang L, Liang Y, Mei J, et al. Family practice and the quality of primary care: a study of Chinese patients in Guangdong Province []]. Fam Pract. 2015;32(5):557.

45. Jesmin S, Thind A, Sarma S. Does team-based primary health care improve patients' perception of outcomes? Evidence from the 2007-08 Canadian Survey of Experiences with Primary Health. [J]. Health Policy. 2012;105(1):71.

46. Zhao Y, Thomas SL, Guthridge SL, et al. Better health outcomes at lower costs: the benefits of primary care utilisation for chronic disease management in remote indigenous communities in Australia's northern territory [J]. BMC Health Serv Res. 2014;14(1):1-9.
47. Nocon RS, Sang ML, Sharma R, et al. Health care use and spending for Medicaid enrollees in federally qualified health centers versus other primary care settings [J]. Am J Public Health. 2016;106(11):e1-9.

48. You Z, Wang L, Feng ZM. Et. Spatial temporal pattern and change characteristics of population distribution in the Pearl River Delta (in Chinese). Trop. Geogr. 2013;33(2):156-63.

49. Yang $H$, Shi L, Lebrun LA, et al. Development of the Chinese primary care assessment tool: data quality and measurement properties [J]. Int. J. Qual. Health Care. 2013;25(1):92.

50. Wang W, Shi L, Yin A, et al. Development and validation of the Tibetan primary care assessment tool [J]. Biomed Res Int. 2014;8:308739.

51. Mei J, Liang $Y$, Shi $L$, et al. The development and validation of a rapid assessment tool of primary Care in China [J]. Biomed Res Int. 2016; 2016(7):1-13.

52 Parchman ML, Burge SK. The patient-physician relationship, primary care attributes, and preventive services. [J]. Fam Med. 2004;36(1):22-7.

53 Cleland JA, Watson MC, Walker L, et al. Community pharmacists' perceptions of barriers to communication with migrants [J]. Int J Pharm Pract. 2012;20(3):148-54.

\section{Ready to submit your research? Choose BMC and benefit from:}

- fast, convenient online submission

- thorough peer review by experienced researchers in your field

- rapid publication on acceptance

- support for research data, including large and complex data types

- gold Open Access which fosters wider collaboration and increased citations

- maximum visibility for your research: over $100 \mathrm{M}$ website views per year

At BMC, research is always in progress.

Learn more biomedcentral.com/submissions 